ISSN 1561-2430 (Print)

ISSN 2524-2415 (Online)

УДК 519.6

Поступила в редакцию 23.12.2020

https://doi.org/10.29235/1561-2430-2021-57-1-14-22

Received 23.12.2020

\author{
Э. А. Айрян ${ }^{1,2}$, М. Гнатич ${ }^{3,4,5}$, В. Б. Малютин ${ }^{6}$ \\ ${ }^{1}$ Лаборатория информационных технологий, \\ Объединенный институт ядерных исследований, Дубна, Россия \\ ${ }^{2}$ Государственный университет «Дубна», Дубна, Россия \\ ${ }^{3}$ Лаборатория теоретической физики им. Н. Н. Боголюбова, \\ Объединенный институт ядерных исследований, Дубна, Россия \\ ${ }^{4}$ Институт экспериментальной физики Словаикой академии наук, Кошице, Словакия \\ ${ }^{5}$ Факультет естествознания, Университет Павла Йозефа Шафарика, Кошище, Словакия \\ ${ }^{6}$ Институт математики Национальной академии наук Беларуси, Минск, Беларусь

\section{ПРИБЛИЖЕННОЕ ВЫЧИСЛЕНИЕ ФУНКЦИОНАЛЬНЫХ ИНТЕГРАЛОВ, ПОРОЖДЕННЫХ УРАВНЕНИЕМ ДИРАКА С ПСЕВДОСПИНОВОЙ СИММЕТРИЕЙ}

Аннотация. Рассматриваются матричнозначные функциональные интегралы, порожденные уравнением Дирака
с релятивистским гамильтонианом. Гамильтониан Дирака содержит скалярный и векторный потенциалы. Сумма
скалярного и векторного потенциалов равна нулю, т. е. случай псевдоспиновой симметрии исследуется. В этом слу-
чае строится уравнение шредингеровского типа на собственные значения и собственные фунции релятивистского
гамильтониана, порождающего функциональный интеграл. Собственные значения и собственные функции операто-
ра шредингеровского типа находятся с помощью метода последовательностей Штурма и метода обратной итерации.
Предлагается метод для вычисления матричнозначных функциональных интегралов специального вида, который
основан на соотношении между функциоальным интегралом и ядром оператора эволюции с релятивистским га-
мильтонианом и на разложении ядра оператора эволюции по найденным собственным функциям релятивистского
гамильтониана.

Ключевые слова: функциональные интегралы, уравнение Дирака, релятивистский гамильтониан, псевдоспиновая симметрия, собственные функции гамильтониана, последовательность Штурма

Для цитирования. Айрян, Э. А. Приближенное вычисление функциональных интегралов, порожденных уравнением Дирака с псевдоспиновой симметрией / Э. А. Айрян, М. Гнатич, В. Б. Малютин // Вес. Нац. акад. навук Беларусі. Сер. фіз.-мат. навук. - 2021. - Т. 57, № 1. - С. 14-22. https://doi.org/10.29235/1561-2430-2021-57-1-14-22

\author{
Edik A. Ayryan ${ }^{1,2}$, Michal Hnatic ${ }^{3,4,5}$, Victor B. Malyutin ${ }^{6}$ \\ ${ }^{1}$ Laboratory of Information Technologies, Joint Institute for Nuclear Research, Dubna, Russia \\ ${ }^{2}$ State University «Dubna», Dubna, Russia \\ ${ }^{3}$ Bogoliubov Laboratory of Theoretical Physics, Joint Institute for Nuclear Research, Dubna, Russia \\ ${ }^{4}$ Institute of Experimental Physics, Slovak academy of Sciences, Košice, Slovak Republic \\ ${ }^{5}$ Faculty of Sciences, P. J. Šafárik University in Košice, Košice, Slovak Republic \\ ${ }^{6}$ Institute of Mathematics of the National Academy of Sciences of Belarus, Minsk, Belarus
}

\title{
APPROXIMATE EVALUATION OF THE FUNCTIONAL INTEGRALS GENERATED BY THE DIRAC EQUATION WITH PSEUDOSPIN SYMMETRY
}

\begin{abstract}
In this paper, the matrix-valued functional integrals generated by the Dirac equation with relativistic Hamiltonian are considered. The Dirac Hamiltonian contains scalar and vector potentials. The sum of the scalar and vector potentials is equal to zero, i.e., the case of pseudospin symmetry is investigated. In this case, a Schrödinger-type equation for the eigenvalues and eigenfunctions of the relativistic Hamiltonian generating the functional integral is constructed. The eigenvalues and eigenfunctions of the Schrödinger-type operator are found using the Sturm sequence method and the reverse iteration method. A method for the evaluation of matrix-valued functional integrals is proposed. This method is based on the relation between the functional integral and the kernel of the evolution operator with the relativistic Hamiltonian and the expansion of the kernel of the evolution operator in terms of the found eigenfunctions of the relativistic Hamiltonian.

Keywords: functional integrals, Dirac equation, relativistic Hamiltonian, pseudospin symmetry, eigenfunctions of Hamiltonian, Sturm sequences
\end{abstract}

(C) Айрян Э. А., Гнатич М., Малютин В. Б., 2021 
For citation. Ayryan E. A., Hnatic M., Malyutin V. B. Approximate evaluation of the functional integrals generated by the Dirac equation with pseudospin symmetry. Vestsi Natsyianal'nai akademii navuk Belarusi. Seryia fizika-matematychnykh navuk = Proceedings of the National Academy of Sciences of Belarus. Physics and Mathematics series, 2021, vol. 57, no. 1, pp. 14-22 (in Russian). https://doi.org/10.29235/1561-2430-2021-57-1-14-22

Введение. Применение теории функциональных интегралов при построении математических моделей, которые используются в квантовой теории поля, статистической механике и стохастическом анализе, приводит к появлению различных типов функциональных интегралов [1-3]. Разнообразие типов функциональных интегралов обусловлено разнообразием способов определения интеграла и областей, где определяется и принимает значения функциональный интеграл $[4,5]$. В данной работе рассматривается функциональный интеграл, порожденный уравнением Дирака с псевдоспиновой симметрией [6]. Это матричнозначный функциональный интеграл, т. е. интеграл, принимающий значения в пространстве матриц. Этот интеграл связан с ядром оператора эволюции, имеющего вид экспоненты с релятивистским гамильтонианом в показателе.

В [7-9] исследовался метод вычисления функциональных интегралов, который основан на разложении по собственным функциям нерелятивистского гамильтониана, входящего в оператор эволюции и порождающего функциональный интеграл. Для нерелятивистского гамильтониана этот метод применим, так как уравнение на собственные значения и собственные функции гамильтониана имеет вид стационарного уравнения Шредингера, для которого можно найти приближенное решение.

Для релятивистского гамильтониана в общем случае не удается получить уравнение на собственные значения и собственные функции гамильтониана в виде стационарного уравнения Шредингера. В [10] получено стационарное уравнение Шредингера для собственных значений и собственных функций при наличии малого параметра $\frac{1}{2 m c^{2}}$. Для нахождения стационарного уравнения Шредингера использовалась аппроксимация, которая получалась разложением в ряд по степеням $\frac{1}{2 m c^{2}}$ с точностью до членов наименьшего порядка.

В настоящей работе рассматривается случай псевдоспиновой симметрии (сумма векторного и скалярного потенциалов равна нулю). В этом случае строится уравнение шредингеровского типа для собственных значений и собственных функций. Затем строится метод для вычисления функциональных интегралов, основанный на разложении по собственным функциям релятивистского гамильтониана, порождающего функциональный интеграл.

В разделе 1 вводится матричнозначный функциональный интеграл, порожденный уравнением Дирака с псевдоспиновой симметрией. Затем строится уравнение шредингеровского типа на собственные значения и собственные функции релятивистского гамильтониана, порождающего интеграл, а также метод для вычисления функциональных интегралов. В разделе 2 приводятся численные результаты. Собственные значения и собственные функции оператора шредингеровского типа находятся с помощью метода последовательностей Штурма и метода обратной итерации.

1. Аналитические результаты. В данном разделе рассматриваются матричнозначные функциональные интегралы, которые определяются на пространстве функций $x(\tau), s \leq \tau \leq t$, удовлетворяющих условию $x(s)=0$. Интегралы определяются равенством

$$
\begin{aligned}
& \int \exp \left\{\int_{s}^{t}[\beta \Phi(x(\tau))+V(x(\tau))] d \tau\right\} \mathrm{d} \mu(x)=
\end{aligned}
$$

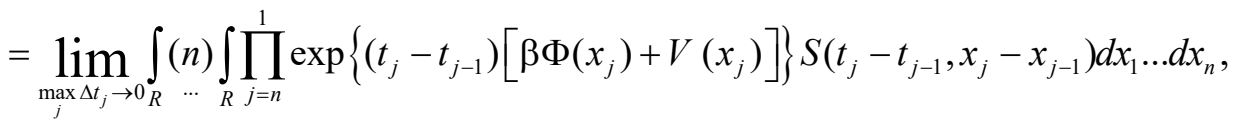

если этот предел существует для любого разбиения отрезка $[s, t]$ точками $s=t_{0}<t_{1}<\ldots<t_{n}=t$.

Здесь $x_{j}=x\left(t_{j}\right), S\left(t_{j}-t_{j-1}, x_{j}-x_{j-1}\right)-$ переходная функция, являющаяся фундаментальным решением уравнения 


$$
\frac{\partial S(t, x)}{\partial t}=a \alpha \frac{\partial S(t, x)}{\partial x}+b \beta S(t, x)
$$

где $a, b$ - вещественные параметры, $\alpha, \beta$ - антикоммутирующие матрицы, т. е. $\alpha \beta+\beta \alpha=0$.

Этот интеграл можно записать в виде $[11,12]$

$$
\int \exp \left\{\int_{s}^{t}[\beta \Phi(x(\tau))+V(x(\tau))] d \tau\right\} \mathrm{d} \mu(x)=\int_{-\infty}^{+\infty} K\left(0, x_{t}\right) d x_{t},
$$

где $K\left(x_{s}, x_{t}\right)$ - ядро оператора эволюции $\exp (t H)$ с релятивистским гамильтонианом $H=a \alpha \frac{\partial}{\partial x}+b \beta+\beta \Phi(x)+V(x)$, соответствующим уравнению Дирака.

Функционал $\Phi(x)$ называется скалярным потенциалом, функционал $V(x)$ называется векторным потенциалом.

Из (3) следует, что для вычисления функционального интеграла можно использовать разложение функции $K\left(x_{s}, x_{t}\right)$ по собственным функциям гамильтониана $H$, порождающего функциональный интеграл.

Чтобы выполнялось условие псевдоспиновой симметрии и антикоммутируемости матриц $\alpha$, $\beta$, будем считать, что

$$
\alpha=\left(\begin{array}{cc}
0 & -1 \\
1 & 0
\end{array}\right), \quad \beta=\left(\begin{array}{cc}
1 & 0 \\
0 & -1
\end{array}\right) .
$$

При сделанных предположениях уравнения для собственных значений $E$ и собственных функций $(\varphi(x), \psi(x))^{T}$ (T- знак транспонирования) оператора $H$ имеют вид

$$
\begin{aligned}
& -a \partial_{x} \psi(x)+b \varphi(x)+\Phi(x) \varphi(x)+V(x) \varphi(x)=E \varphi(x), \\
& a \partial_{x} \varphi(x)-b \psi(x)-\Phi(x) \psi(x)+V(x) \psi(x)=E \psi(x),
\end{aligned}
$$

где $\partial_{x}-$ производная по переменной $x$.

При построении уравнения шредингеровского типа для собственных значений и собственных функций мы рассматриваем случай псевдоспиновой симметрии [6], т. е. $\Phi(x)+V(x)=0$. Тогда уравнения (4) примут вид

$$
\begin{gathered}
a \partial_{x} \psi(x)-(b-E) \varphi(x)=0, \\
a \partial_{x} \varphi(x)+(-b+2 V(x)-E) \psi(x)=0 .
\end{gathered}
$$

Из этих равенств получаем уравнение для $\psi(x)$ :

$$
\left(\partial_{x}^{2}+\frac{(b-E) 2 V(x)}{a^{2}}-\frac{b^{2}-E^{2}}{a^{2}}\right) \psi(x)=0 .
$$

Функция $\varphi(x)$ выражается через функцию $\psi(x)$ равенством

$$
\varphi(x)=\frac{a}{b-E} \partial_{x} \psi(x) .
$$

Чтобы найти $c$ и $\psi_{j}(x)$, удовлетворяющие уравнению (5), с помощью метода последовательностей Штурма [13] находим приближенное собственное значение $\lambda_{j}$ оператора

$$
\partial_{x}^{2}+\frac{\left(b-E_{j}\right) 2 V(x)}{a^{2}} .
$$


Вычисляем $E_{j}^{\prime}=-\sqrt{b^{2}-\lambda_{j} a^{2}}$. Подбираем $E_{j}$ так, чтобы $E_{j} \approx E_{j}^{\prime}$ с заданной точностью. Эти $E_{j}$ и выбираем в качестве приближенных собственных значений. Затем методом обратной итерации [13] находим собственные векторы $\psi_{j}(x)$.

Используя равенство (6), находим функции $\left(\varphi_{j}(x), \psi_{j}(x)\right)^{T}$, являющиеся собственными функциями оператора $H$ с собственными значениями $E_{j}$.

Векторы $\left(\varphi_{j}(x), \psi_{j}(x)\right)^{T}$, вообще говоря, не ортогональны при различных значениях $j$. Для их ортогонализации можно использовать процесс Грама - Шмидта. При этом ортогональные векторы строятся по правилу

$$
\begin{gathered}
\eta_{0}=\left(\varphi_{0}(x), \psi_{0}(x)\right)^{T}, \\
\eta_{j}=\left(\varphi_{j}(x), \psi_{j}(x)\right)^{T}-\sum_{k=0}^{j-1} \frac{\left\langle\left(\varphi_{j}(x), \psi_{j}(x)\right)^{T}, \eta_{k}\right\rangle}{\left\langle\eta_{k}, \eta_{k}\right\rangle} \eta_{k},
\end{gathered}
$$

где

$$
\left\langle\eta_{k}, \eta_{k}\right\rangle=\left\langle\left(\varphi_{k}(x), \psi_{k}(x)\right)^{T},\left(\varphi_{k}(x), \psi_{k}(x)\right)^{T}\right\rangle=\int_{-\infty}^{+\infty} \varphi_{k}(x) \varphi_{k}(x) d x+\int_{-\infty}^{+\infty} \psi_{k}(x) \psi_{k}(x) d x .
$$

Для разложения ядра оператора $\exp (t H)$ по векторам $\eta_{j}$ можно использовать формулу

$$
K\left(x_{s}, x_{t}\right)=\sum_{j=0}\left\langle\eta_{j}, \eta_{j}\right\rangle^{-1}\left[\exp (t H) \eta_{j}\right]\left(x_{t}\right) \eta_{j}^{T}\left(x_{s}\right)
$$

Из этой формулы и формулы (3) получаем выражение для функционального интеграла

$$
\int \exp \left\{\int_{s}^{t}[\beta \Phi(x(\tau))+V(x(\tau))] d \tau\right\} \mathrm{d} \mu(x)=\sum_{j=0}^{+\infty} \int_{-\infty}^{\langle}\left\langle\eta_{j}, \eta_{j}\right\rangle^{-1}\left[\exp (t H) \eta_{j}\right]\left(x_{t}\right) \eta_{j}^{T}(0) d x_{t} .
$$

Так как $\eta_{j}$ выражаются через собственные векторы оператора $H$, то $\exp (t H) \eta_{j}$ может быть записано через $\exp \left(t E_{k}\right)$ и $\left(\varphi_{j}(x), \psi_{j}(x)\right)^{T}, 0 \leq k \leq j$.

Таким образом, мы получаем приближенную формулу для вычисления функционального интеграла с помощью собственных значений $E_{j}, j=0,1,2, \ldots$, и собственных функций $\left(\varphi_{j}(x), \psi_{j}(x)\right)^{T}$ гамильтониана $H$.

2. Численные результаты. Рассмотрим применение предложенного метода к вычислению функционального интеграла (3) где

$$
V(x)=-\frac{x^{2}}{2}, \quad \Phi(x)=\frac{x^{2}}{2} .
$$

Если в формуле (8) рассмотреть только слагаемое с $j=0$, то получим

$$
\begin{gathered}
\int \exp \left\{\int_{s}^{t}[\beta \Phi(x(\tau))+V(x(\tau))] d \tau\right\} \mathrm{d} \mu(x) \approx \int_{-\infty}^{+\infty}\left\langle\eta_{0}, \eta_{0}\right\rangle^{-1}\left[\exp (t H) \eta_{0}\right]\left(x_{t}\right) \eta_{0}^{T}(0) d x_{t}, \\
\eta_{0}=\left(\varphi_{0}(x), \psi_{0}(x)\right)^{T} .
\end{gathered}
$$

Поэтому это приближенное равенство можно переписать в виде 


$$
\begin{aligned}
& \int \exp \left\{\int_{s}^{t}[\beta \Phi(x(\tau))+V(x(\tau))] d \tau\right\} \mathrm{d} \mu(x) \approx \\
& \approx\left(\int_{-\infty}^{+\infty} \varphi_{0}^{2}(x) d x+\int_{-\infty}^{+\infty} \psi_{0}^{2}(x) d x\right)^{-1} \exp \left(t E_{0}\right)\left(\begin{array}{ll}
\int_{-\infty}^{+\infty} \varphi_{0}\left(x_{t}\right) d x_{t} \varphi_{0}(0) & \int_{-\infty}^{+\infty} \varphi_{0}\left(x_{t}\right) d x_{t} \psi_{0}(0) \\
\int_{-\infty}^{+\infty} \psi_{0}\left(x_{t}\right) d x_{t} \varphi_{0}(0) & \int_{-\infty}^{+\infty} \psi_{0}\left(x_{t}\right) d x_{t} \psi_{0}(0)
\end{array}\right) .
\end{aligned}
$$

При $s=0, t=1, a=1, b=1, A=5$ ([- $A, A]$ - интервал, на котором рассматриваются приближенные собственные функции), $N=90$ ( $N$ - количество интервалов, на которое делится интервал $[-A, A]$ для приближенного вычисления собственных значений и собственных функций)

$$
\begin{aligned}
E_{0} \approx-1,617 ; \quad \int_{-\infty}^{+\infty} \psi_{0}\left(x_{t}\right) d x_{t} \approx & 1,669 ; \quad \int_{-\infty}^{+\infty} \varphi_{0}\left(x_{t}\right) d x_{t} \approx 0 ; \quad \psi_{0}(0) \approx 0,8478 ; \quad \varphi_{0}(0) \approx-0,0292 ; \\
& \int_{-\infty}^{+\infty} \varphi_{0}^{2}(x) d x+\int_{-\infty}^{+\infty} \psi_{0}^{2}(x) d x \approx 1,1181 .
\end{aligned}
$$

Таким образом,

$$
\int \exp \left\{\int_{s}^{t}[\beta \Phi(x(\tau))+V(x(\tau))] d \tau\right\} \mathrm{d} \mu(x) \approx\left(\begin{array}{cc}
0 & 0 \\
-0,0087 & 0,2512
\end{array}\right) .
$$

Теперь вычислим вклад, который дает слагаемое с $j=1$ в формуле (8). В данном случае вектор $\left(\varphi_{1}(x), \psi_{1}(x)\right)^{T}$ ортогонален вектору $\left(\varphi_{0}(x), \psi_{0}(x)\right)^{T}$. Поэтому $\eta_{1}=\left(\varphi_{1}(x), \psi_{1}(x)\right)^{T}$. Следовательно, слагаемое с $j=1$ в формуле (8) можно записать в виде

$$
\left(\int_{-\infty}^{+\infty} \varphi_{1}^{2}(x) d x+\int_{-\infty}^{+\infty} \psi_{1}^{2}(x) d x\right)^{-1} \exp \left(t E_{1}\right)\left(\begin{array}{ll}
\int_{-\infty}^{+\infty} \varphi_{1}\left(x_{t}\right) d x_{t} \varphi_{1}(0) & \int_{-\infty}^{+\infty} \varphi_{1}\left(x_{t}\right) d x_{t} \psi_{1}(0) \\
\int_{-\infty}^{+\infty} \psi_{1}\left(x_{t}\right) d x_{t} \varphi_{1}(0) & \int_{-\infty}^{+\infty} \psi_{1}\left(x_{t}\right) d x_{t} \psi_{1}(0)
\end{array}\right) .
$$

При $s=0, t=1, a=1, b=1, A=5, N=90$

$$
\begin{gathered}
E_{1} \approx-2,581 ; \quad \int_{-\infty}^{+\infty} \psi_{1}\left(x_{t}\right) d x_{t} \approx 10^{-5} ; \quad \int_{-\infty}^{+\infty} \varphi_{1}\left(x_{t}\right) d x_{t} \approx 0 ; \quad \psi_{1}(0) \approx 4 \cdot 10^{-6} ; \quad \varphi_{1}(0) \approx-0,4753 ; \\
\int_{-\infty}^{+\infty} \varphi_{1}^{2}(x) d x+\int_{-\infty}^{+\infty} \psi_{1}^{2}(x) d x \approx 1,5745 .
\end{gathered}
$$

Таким образом, слагаемое с $j=1$ дает вклад

$$
\left(\begin{array}{cc}
0 & 0 \\
-2 \cdot 10^{-7} & 2 \cdot 10^{-12}
\end{array}\right)
$$

Теперь вычислим вклад, который дает слагаемое с $j=2$ в формуле (8). Вектор $\left(\varphi_{2}(x), \psi_{2}(x)\right)^{T}$ ортогонален вектору $\left(\varphi_{1}(x), \psi_{1}(x)\right)^{T}$, но не ортогонален вектору $\left(\varphi_{0}(x), \psi_{0}(x)\right)^{T}$. Поэтому $\eta_{2}=\left(\eta_{2,1}, \eta_{2,2}\right)^{T}=\left(\varphi_{2}(x), \psi_{2}(x)\right)^{T}+0,081 \cdot\left(\varphi_{0}(x), \psi_{0}(x)\right)^{T}$. Следовательно, слагаемое с $j=2$ в формуле (8) можно записать в виде 


$$
\begin{aligned}
& \left(\int_{-\infty}^{+\infty} \eta_{2,1}^{2}(x) d x+\int_{-\infty}^{+\infty} \eta_{2,2}^{2}(x) d x\right)^{-1} \exp \left(t E_{2}\right)\left(\begin{array}{ll}
\int_{-\infty}^{+\infty} \varphi_{2}\left(x_{t}\right) d x_{t} \eta_{2,1}(0) & \int_{-\infty}^{+\infty} \varphi_{2}\left(x_{t}\right) d x_{t} \eta_{2,2}(0) \\
\int_{-\infty}^{+\infty} \psi_{2}\left(x_{t}\right) d x_{t} \eta_{2,1}(0) & \int_{-\infty}^{+\infty} \psi_{2}\left(x_{t}\right) d x_{t} \eta_{2,2}(0)
\end{array}\right)+ \\
& +0,081 \cdot\left(\int_{-\infty}^{+\infty} \eta_{2,1}^{2}(x) d x+\int_{-\infty}^{+\infty} \eta_{2,2}^{2}(x) d x\right)^{-1} \exp \left(t E_{0}\right)\left(\begin{array}{ll}
\int_{-\infty}^{+\infty} \varphi_{0}\left(x_{t}\right) d x_{t} \eta_{2,1}(0) & \int_{-\infty}^{+\infty} \varphi_{0}\left(x_{t}\right) d x_{t} \eta_{2,2}(0) \\
\int_{-\infty}^{+\infty} \psi_{0}\left(x_{t}\right) d x_{t} \eta_{2,1}(0) & \int_{-\infty}^{+\infty} \psi_{0}\left(x_{t}\right) d x_{t} \eta_{2,2}(0)
\end{array}\right) .
\end{aligned}
$$

При $s=0, t=1, a=1, b=1, A=5, N=90$

$$
\begin{gathered}
E_{2} \approx-3,379 ; \quad \int_{-\infty}^{+\infty} \psi_{2}\left(x_{t}\right) d x_{t} \approx-1,1035 ; \quad \int_{-\infty}^{+\infty} \varphi_{2}\left(x_{t}\right) d x_{t} \approx 0 ; \quad \psi_{2}(0) \approx 0,6421 ; \quad \varphi_{2}(0) \approx-0,0849 ; \\
\int_{-\infty}^{+\infty} \eta_{2,1}^{2}(x) d x+\int_{-\infty}^{+\infty} \eta_{2,2}^{2}(x) d x \approx 1,2655 ; \quad \eta_{2,1}(0) \approx-0,0873 ; \quad \eta_{2,2}(0) \approx 0,7108 .
\end{gathered}
$$

Таким образом, слагаемое с $j=2$ дает вклад

$$
\left(\begin{array}{cc}
0 & 0 \\
0,0007 & -0,006
\end{array}\right)
$$

С учетом вклада трех слагаемых получаем

$$
\int \exp \left\{\int_{s}^{t}[\beta \Phi(x(\tau))+V(x(\tau))] d \tau\right\} \mathrm{d} \mu(x) \approx\left(\begin{array}{cc}
0 & 0 \\
-0,0080 & 0,2452
\end{array}\right) .
$$

Для элементов матрицы

$$
\int \exp \left\{\int_{s}^{t}[\beta \Phi(x(\tau))+V(x(\tau))] d \tau\right\} \mathrm{d} \mu(x)
$$

с индексами $(1,2)$ и $(2,1)$ при $V(x)=-\frac{x^{2}}{2}, \Phi(x)=\frac{x^{2}}{2}$ известны точные значения и они равны нулю (см. Приложение). Для сравнения на рисунке приведены приближенные значения элементов матричнозначного интеграла с индексом $(2,1)$ для различных значений $N$.

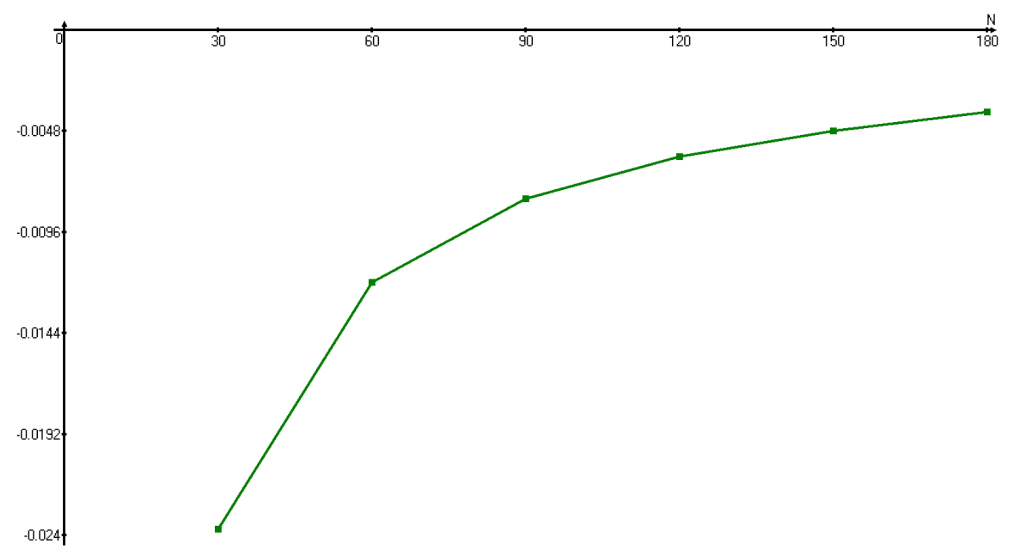

Приближенные значения элемента матричнозначного интеграла с индексом $(2,1), s=0, t=1, a=1, b=1, A=5$

Approximate values of the element of the matrix-valued integral with index $(2,1), s=0, t=1, a=1, b=1, A=5$ 
Из рисунка видно, что приближенные значения элементов матричнозначного интеграла стремятся к точному значению при увеличении количества интервалов $N$, на которое делится интервал $[-A, A]$ для приближенного вычисления собственных значений и собственных функций.

Благодарности. Исследование выполнено при финансовой поддержке Российского фонда фундаментальных исследований в рамках научного проекта № 19-01-00645.
Acknowledgements. The research was carried out under the financial support of the Russian Foundation for Basic Research within the framework of Project no. 19-01-00645.

\section{Список использованных источников}

1. Glimm, J. Quantum Physics. A Functional Integral Point of View / J. Glimm, A. Jaffe. - Berlin; Heidelberg; New York: Springer-Verlag 1981. - 417 p.

2. Kleinert, H. Path Integrals in Quantum Mechanics, Statistics, Polymer Physics, and Financial Markets / H. Kleinert. Singapore: World Scientific Publishing, 2004. - 1504 p. https://doi.org/10.1142/5057

3. Feynman, R. P. Quantum Mechanics and Path Integrals. / R. P. Feynman, A. R. Hibbs. - New York: McGraw-Hill, 1965. $-382 \mathrm{p}$.

4. Egorov, A. D. Functional Integrals: Approximate Evaluation and Applications / A. D. Egorov, P. I. Sobolevsky, L. A. Yanovich. - Dordrecht: Kluwer Academic Pabl., 1993. - 400 p. https://doi.org/10.1007/978-94-011-1761-6

5. Егоров, А. Д. Введение в теорию и приложения функционального интегрирования / А. Д. Егоров, Е. П. Жидков, Ю. Ю. Лобанов. - М.: Физматлит, 2006. - 400 с.

6. Berkdemir, C. Pseudospin symmetry solution of the Dirac equation with an angle-dependent potential / C. Berkdemir, R. Sever // J. Phys. A: Mathematical and Theoretical. - Vol. 41, № 4. - P. 045302.https://doi.org/10.1088/1751-8113/41/4/045302

7. Малютин, В. Б. Вычисление функциональных интегралов с помощью последовательностей Штурма / В. Б. Малютин // Вес. Нац. акад. Навук Беларусі. Сер. фіз.-мат. навук. - 2016. - № 4. - С. 32-37.

8. Малютин, В. Б. О вычислении функциональных интегралов, порожденных некоторыми нерелятивистскими гамильтонианами / В. Б. Малютин // Вес. Нац. акад. Навук Беларусі. Сер. фіз.-мат. навук. - 2018. - Т. 54, № 1. - С. $44-49$.

9. Малютин, В. Б. Приближенное вычисление функциональных интегралов, содержащих центробежный потенциал / В. Б. Малютин // Вес. Нац. акад. Наву кБеларусі. Сер. фіз.-мат. навук. - 2019. - Т. 55, № 2. - C. 152-157. https:// doi.org/10.29235/1561-2430-2019-55-2-152-157

10. Айрян, Э. А. Приближенное вычисление функциональных интегралов, порожденных релятивистским гамильтонианом / Э. А. Айрян, М. Гнатич, В. Б. Малютин // Вес. Нац. акад. навук Беларусі. Сер.фіз.-мат. навук. - 2020. T. 56, № 1. - C. 72-83. https://doi.org/10.29235/1561-2430-2020-56-1-72-83

11. Ichinose, T. Propagation of a Dirac particle. A path integral approach / T. Ichinose, H. Tamura // J. Math. Phys. 1984. - Vol. 25, № 6. - P. 1810-1819.https://doi.org/10.1063/1.526360

12. Ichinose, T. The zitterbewegung of a Dirac particle in two-dimensional space-time / T. Ichinose, H. Tamura // J. Math. Phys. - 1988. - Vol. 29, № 1. - P. 103-109. https://doi.org/10.1063/1.528162

13. Wilkinson, J. H. The Algebraic Eigenvalue Problem / J. H. Wilkinson. - Oxford, 1965. - 662 p.

\section{References}

1. Glimm J., Jaffe A. Quantum Physics. A Functional Integral Point of View. Berlin, Heidelberg, New York, SpringerVerlag, 1981. 417 p.

2. Kleinert H. Path Integrals in Quantum Mechanics, Statistics, Polymer Physics, and Financial Markets. Singapore, World Scientific Publishing, 2004. 1504 p. https://doi.org/10.1142/5057

3. Feynman R. P., Hibbs A. R. Quantum Mechanics and Path Integrals. New York, McGraw-Hill, 1965. 382 p.

4. Egorov A. D., Sobolevsky P. I., Yanovich L. A. Functional Integrals: Approximate Evaluation and Applications. Dordrecht, Kluwer Academic Pabl., 1993. 400 p. https://doi.org/10.1007/978-94-011-1761-6

5. Egorov A. D., Zhidkov E. P., Lobanov Yu. Yu. Introduction to Theory and Applications of Functional Integration. Moscow, Fizmatlit Publ., 2006. 400 p. (in Russian).

6. Berkdemir C., Sever R. Pseudospin symmetry solution of the Dirac equation with an angle-dependent potential. Journal of Physics A: Mathematical and Theoretical, 2008, vol. 41, no. 4, pp. 045302. https://doi.org/10.1088/1751-8113/41/4/045302

7. Malyutin V. B. Evaluation of functional integrals using Sturm sequences. Vestsi Natsyianal'nai akademii navuk Belarusi. Seryia fizika-matematychnykh navuk = Proceedings of the National Academy of Sciences of Belarus. Physics and Mathematics series, 2016, no. 4, pp. 32-37 (in Russian).

8. Malyutin V. B. Evaluation of functional integrals generated by some nonrelativistic Hamiltonians. Vestsi Natsyianal'nai akademii navuk Belarusi. Seryia fizika-matematychnykh navuk = Proceedings of the National Academy of Sciences of Belarus. Physics and Mathematics series, 2018, vol. 54, no.1, pp. $44-49$ (in Russian).

9. Malyutin V. B. Approximate evaluation of functional integrals with centrifugal potential. Vestsi Natsyianal'nai akademii navuk Belarusi. Seryia fizika-matematychnykh navuk = Proceedings of the National Academy of Sciences of Belarus. Physics and Mathematics series, 2019, vol. 55, no. 2, pp. 152-157 (in Russian). https://doi.org/10.29235/1561-24302019-55-2-152-157 
10. Ayryan E. A., Hnatic M., Malyutin V. B. Approximate evaluation of functional integrals generated by the relativistic Hamiltonian. Vestsi Natsyianal'nai akademii navuk Belarusi.S eryia fizika-matematychnykh navuk = Proceedings of the National Academy of Sciences of Belarus. Physics and Mathematics series, 2020, vol. 56, no. 1, pp. $72-83$ (in Russian). https://doi.org/10.29235/1561-2430-2020-56-1-72-83

11. Ichinose T., Tamura H. Propagation of a Dirac particle. A path integral approach. Journal of Mathematics and Physics, 1984, vol. 25, no. 6, pp. 1810-1819. https://doi.org/10.1063/1.526360

12. Ichinose T., Tamura H. The zitterbewegung of a Dirac particle in two-dimensional space-time. Journal of Mathematics and Physics, 1988, vol. 29, no. 1, pp. 103-109. https://doi.org/10.1063/1.528162

13. Wilkinson J. H. The Algebraic Eigenvalue Problem. Oxford, 1965. 662 p.

Приложение

В данном приложении рассматривается точное вычисление элементов матрицы

$$
I=\int \exp \left\{\int_{s}^{t}[\beta \Phi(x(\tau))+V(x(\tau))] d \tau\right\} \mathrm{d} \mu(x)
$$

с индексами $(1,2)$ и $(2,1)$ при $V(x)=-\frac{x^{2}}{2}, \Phi(x)=\frac{x^{2}}{2}$.

По определению

$$
I=\lim _{\max \Delta t_{j} \rightarrow 0_{R}} \int_{\ldots}(n) \int_{R} \prod_{j=n}^{1}\left[\exp \left\{\left(t_{j}-t_{j-1}\right)\left[\beta \frac{x_{j}^{2}}{2}-\frac{x_{j}^{2}}{2}\right]\right\} S\left(t_{j}-t_{j-1}, x_{j}-x_{j-1}\right)\right] d x_{1} \ldots d x_{n},
$$

где $\alpha=\left(\begin{array}{cc}0 & -1 \\ 1 & 0\end{array}\right), \beta=\left(\begin{array}{cc}1 & 0 \\ 0 & -1\end{array}\right)$.

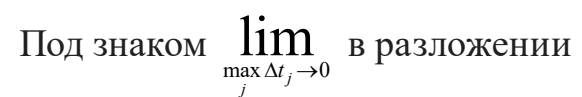

$$
\exp \left\{\left(t_{j}-t_{j-1}\right)\left[\beta \frac{x_{j}^{2}}{2}-\frac{x_{j}^{2}}{2}\right]\right\}
$$

в ряд можно оставить только слагаемые с $t_{j}-t_{j-1}$ в нулевой и первой степени. Получим

$$
I=\lim _{\substack{\max _{j} \Delta t_{j} \rightarrow 0_{R} \\ \int_{R}}}(n) \int_{R}^{1}\left[\left(1+\frac{\left(t_{j}-t_{j-1}\right) \beta x_{j}^{2}}{2}\right)\left(1-\frac{\left(t_{j}-t_{j-1}\right) x_{j}^{2}}{2}\right) S\left(t_{j}-t_{j-1}, x_{j}-x_{j-1}\right)\right] d x_{1} \ldots d x_{n} .
$$

После замены переменных $x_{j}-x_{j-1}=y_{j}, 1 \leq j \leq n$, получим

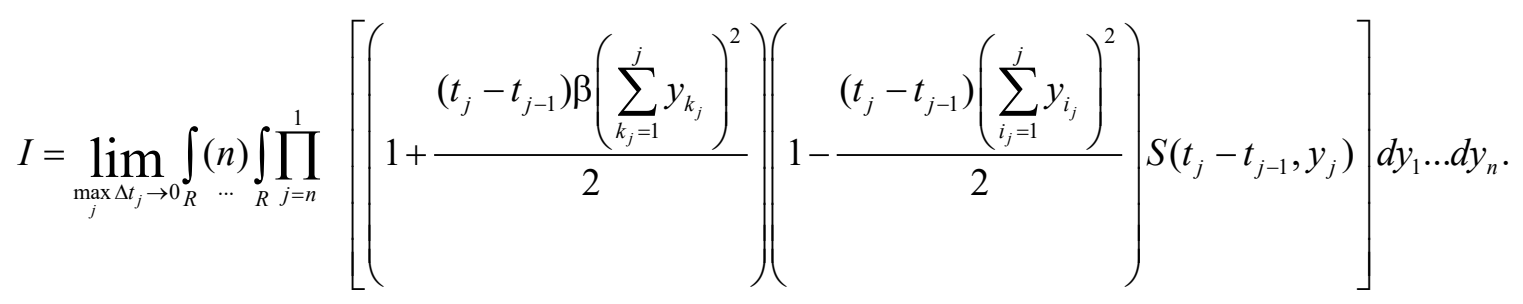

Из этого равенства следует, что интеграл можно представить в виде суммы, слагаемые которой имеют вид

$$
c \int_{R}(n) \int_{R} \prod_{j=n}^{1}\left[\beta^{e_{j}} y_{j}^{d_{j}} S\left(t_{j}-t_{j-1}, y_{j}\right)\right] d y_{1} \ldots d y_{n},
$$

где $c$ - некоторая константа, $e_{j}$ принимают значения 0 или $1, d_{j}$ принимают целые значения, $0 \leq d_{j} \leq 2(n+1-j), \sum_{j=1}^{n} d_{j}$ принимает четные значения. 
Для $S\left(t_{j}-t_{j-1}, y_{j}\right)$ верно равенство

$$
S\left(t_{j}-t_{j-1}, y_{j}\right)=\frac{1}{2 \pi} \int \exp \left\{\left(t_{j}-t_{j-1}\right)\left(-i a \alpha z_{j}+b \beta\right)\right\} \exp \left\{-i y_{j} z_{j}\right\} d z_{j} .
$$

Отсюда при малых $t_{j}-t_{j-1}$ для $S\left(t_{j}-t_{j-1}, y_{j}\right)$ получаем приближенное равенство

$$
\begin{gathered}
S\left(t_{j}-t_{j-1}, y_{j}\right) \approx \frac{1}{2 \pi} \int\left[\cosh \left(-\left(t_{j}-t_{j-1}\right) i a z_{j}\right)+\alpha \sinh \left(-\left(t_{j}-t_{j-1}\right) i a z_{j}\right)\right] \exp \{b \beta\} \exp \left\{-i y_{j} z_{j}\right\} d z_{j}= \\
=\frac{1}{2}\left[\delta\left(\left(t_{j}-t_{j-1}\right) a+y_{j}\right)+\delta\left(-\left(t_{j}-t_{j-1}\right) a+y_{j}\right)+\alpha \delta\left(\left(t_{j}-t_{j-1}\right) a+y_{j}\right)-\alpha \delta\left(-\left(t_{j}-t_{j-1}\right) a+y_{j}\right)\right] \exp \{b \beta\},
\end{gathered}
$$

где $\delta(y)$ - дельта-функция Дирака.

Следовательно,

$$
\begin{gathered}
\int_{R} y_{j}^{d_{j}} S\left(t_{j}-t_{j-1}, y_{j}\right) d y_{j}= \\
=\frac{1}{2}\left[\left(-\left(t_{j}-t_{j-1}\right) a\right)^{d_{j}}+\left(\left(t_{j}-t_{j-1}\right) a\right)^{d_{j}}+\alpha\left(-\left(t_{j}-t_{j-1}\right) a\right)^{d_{j}}-\alpha\left(\left(t_{j}-t_{j-1}\right) a\right)^{d_{j}}\right] \exp \{b \beta\}= \\
=\left(-\alpha\left(t_{j}-t_{j-1}\right) a\right)^{d_{j}} \exp \{b \beta\} .
\end{gathered}
$$

Таким образом, слагаемые (П1) можно записать в виде

$$
c \prod_{j=n}^{1}\left[\beta^{e_{j}}\left(-\alpha\left(t_{j}-t_{j-1}\right) a\right)^{d_{j}} \exp \{b \beta\}\right] .
$$

Так как $\sum_{j=1}^{n} d_{j}$ принимает четные значения и $\alpha^{2}, \beta$ - диагональные матрицы, то матрица в (П2) является диагональной. Следовательно, интеграл $I$ также диагональная матрица, так как он записывается в виде суммы матриц вида (П2). Таким образом, $I_{21}=I_{12}=0$.

\section{Информация об авторах}

Айрян Эдик Арташевич - кандидат физико-математических наук, заведующий сектором, Лаборатория информационных технологий, Объединенный институт ядерных исследований (ул. Жолио-Кюри, 6, 141980, г. Дубна, Российская Федерация); Государственный университет «Дубна» (ул. Университетская, 19, 141980, г. Дубна, Российская Федерация). E-mail: ayrjan@jinr.ru

Гнатич Михал - доктор физико-математических наук наук, профессор, заместитель директора, Лаборатория теоретической физики им. Н. Н. Боголюбова, Объединенный институт ядерных исследований (ул. Жолио-Кюри, 6, 141980, г. Дубна, Российская Федерация); Институт экспериментальной физики Словацкой академии наук (ул. Ватсонова, 47, 040 01, г. Кошице, Словацкая Республика,); Факультет естествознания, Университет Павла Йозефа Шафарика (Парк Ангелинум, 9, 040 01, г. Кошице, Словацкая Республика, ). E-mail: hnatic@saske.sk

Малютин Виктор Борисович - доктор физикоматематических наук, главный научный сотрудник, Институт математики Национальной академии наук Беларуси (ул. Сурганова, 11, 220072, г. Минск, Республика Беларусь). E-mail: malyutin@im.bas-net.by

\section{Information about the authors}

Edik A. Ayryan - Ph. D. (Physics and Mathematics), Head of Sector, Laboratory of Information Technologies, Joint Institute for Nuclear Research (6, Joliot-Curie Str., 141980, Dubna, Russian Federation); State University «Dubna» (19, Universitetskaja Str., 141980, Dubna, Russian Federation). E-mail: ayrjan@jinr.ru

Michal Hnatic - Dr. Sc. (Physics and Mathematics), Professor, Deputy Director, Bogoliubov Laboratory of Theoretical Physics, Joint Institute for Nuclear Research (6, Joliot-Curie Str., Dubna, Russian Federation); Institute of Experimental Physics Slovak Academy of Sciences (IEP SAS) (47, Watsonova Str., Košice, Slovak Republic); Faculty of Science P. J. Safarik University (9, Park Angelinum, Košice, Slovak Republic). E-mail: hnatic@saske.sk

Victor B. Malyutin - Dr. Sc. (Physics and Mathematics), Principal Researcher, Institute of Mathematics of the National Academy of Sciences of Belarus (11, Surganov Str., 220072, Minsk, Republic of Belarus). E-mail: malyutin@ im.bas-net.by 